International Journal of Engineering \& Technology, $7(4.5)(2018) 753-758$
SPC
International Journal of Engineering \& Technology
Website $\frac{w w w . s c i e n c e p u b c o . c o m / i n d e x . p h p / I J E T}{2}$
Research paper

\title{
Server-based positioning system using Bluetooth low energy technology for tracking assets in hospitals
}

\author{
Diogo Brito $^{1}$ *, Lionel Leiva-Marcon ${ }^{2}$, Sara Paiva ${ }^{3}$ \\ ${ }^{1}$ Polytechnic Institute of Viana do Castelo Av. do Atlântico, Viana do Castelo, Portugal Lionel Leiva-Marcon \\ ${ }^{2}$ Proactive Swiss Route de Saint - Cergue 303 Nyon, Swiss \\ ${ }^{3}$ Polytechnic Institute of Viana do Castelo Av. do Atlântico, Viana do Castelo, Portugal \\ *Corresponding author E-mail: diogo.gomes.brito@gmail.com
}

\begin{abstract}
In this paper we present a server-based positioning system to track assets in a hospital environment, using Bluetooth low energy technology. The need to easily and quickly find equipment is undeniable and can sometimes be a life or death situation. We present the system architecture and the components we rely on to calculate distance and equipment coordinates. The main purpose of this solution is to allow medical personnel to find an equipment through a desktop computer in the hospital floor. We also present tests made with several materials that are present in a hospital environment and that can influence the signal.
\end{abstract}

Keywords: Real-Time Location Systems; Indoor Tracking; Assets Tracking and Monitoring; Healthcare Improvement.

\section{Introduction}

Real-time location systems (RTLS) are becoming much more used since wireless technologies provide the necessary infrastructure to develop useful services. RTLS refers to a general area of technology that determines the current position of an objet based on realtime information gathered through a wireless system of some sort [1]. The global positioning system (GPS) was one of the first cases of RTLS to track vehicles and can be used in outdoor environment in scenarios such as logistics applications, transportation management or land-based transports. But not all assets can be tracked the same way as it depends in its nature and location. As indoor environment changes radically the technologies that can be used and almost excludes GPS and cellular based systems due to their lack of signal. Other technologies arise for indoor scenarios such as Radio-frequency identification (RFID), Wi-Fi or, more recently, Bluetooth Low Energy (BLE) technology. Some existent scenarios that use some of these technologies include equipment tracking, real time personnel finding, patient and guest finding [2], among others. So far we spoke about RTLS that track moving assets and the finder can be standing still. But, in fact, the scenario can be the other way around which is very tipical. Let us consider a guided visit to a museum. Beacons can be placed near art pieces (that do not move) and the visitour moves around the museum and can receive in his mobile application information of the art pieces that are more close to him. This is also a RTLS system but beacons are used in a different way and for a different purpose. These two perspetives are called server-based and client-based RTLS systems, respectively.

In this paper, we will focus our study on tracking assets in a hospital scenario so our system will be server-based. The need to track assets is undeniable and we feel that every day when we loose a simple thing - such as our keys or wallet - and would really appreciate that we could simple track them and know where we left it. In this scenario, there is the need to find the asset but not the urgency.
That is not true in a hospital environment which is clearly understand if we think the consequences that can arise of taking 5 minutes to find a reanimation machine.

An important aspect to bear in mind when developing such a system is the type of materials walls are made of as they have a big influence on beacons and the overall performance of the system can be influenced and compromised. This aspect was mentioned in [3] has a problem that could compromise the success of RTLS in hospital environments.

We provide in this paper two main contributions: the architecture and configuration of a system to track assets indoor in real time and a detailed evaluation of several scenarios to prove the technology works no matter the materials involved.

The rest of this paper is organized as follows: in the next section we present some literature review including used technologies in these scenarios and what currently exists in RTLS in hospitals. Next we present our architecture and explain the algorithms that allow to calculate distances and position of equipments. Before conclusions, we present the evaluation of the system, considering the several materials that can affect its behavior.

\section{Literature review}

a) Technologies

In this section, we provide a brief detail on the technologies than can be used for the purpose of a RTLS and also a literature review regarding RTLS applied to hospitals.

1) Radio-frequency identification - RFID

RFID is a technology that uses electromagnetic fields to identify and track objects. A typical RFID systems works with three elements: a scanning antenna, a transceiver and the RFID tag. The antenna scans for tags, and when the tag detects the activation signal, send the information. The RFID technology is more used to simulate bar codes and can be used to track several things such as materials or even animals (and maybe one day humans). This tech- 
nology is not the best for indoor location because it doesn't give us a precise location of the tag, it can only say where the object is in an area. In addition, there are some considerable costs associated when configuring a system because a lot of antennas are required.

2) $\mathrm{Wi}-\mathrm{Fi}$

Wi-Fi positioning is already used in the real life in addition to the GPS system. It works on a typical system of RSSI values. It could be a good technology to use for indoor location because Wi-Fi is present in almost every place, but can represent an elevate cost considering the necessity of having a big number of routers. It is not easy to get a precise indoor location and adding, removing or simply changing the location of a router/AP has a direct impact on the RSSI map.

3) Bluetooth Low Energy - BLE

Bluetooth is a technology present and known by us for many years now. Bluetooth evolved, and the BLE technology appeared. Known also as Bluetooth Smart, it was introduced in 2006 by Nokia under the name of Wibree and merged in 2010 with Bluetooth 4.0 and get the name has we know it today. Beacons are an extension of the BLE technology. In difference with conventional Bluetooth, BLE broadcasts much smaller packets of data and can operate on a logic board with a coin battery on it and it's very cheap to produce or buy. Nowadays, depending on which beacons we use, we can choose between packets to broadcast the data: iBeacon and Eddystone. Although they are almost the same because both broadcasts the same information, the Eddystone packets have an advantage: a telemetry packet is broadcasted too that can give us some additional information, such as the battery voltage or the temperature.

b) RTLS in Hospitals

RTLS is being used in several hospitals such as the Florida Hospital for two main purposes: event alerts and customer satisfaction [4]. In the first case, RTLS contributes to adding context to the patients pathway, trending and predictive modeling, actionable data at the right time to the right person. For customers, it provides real time access, information and flow - engagement in care process, alerts for wait states, knowledge of their progress in their care. Another study on RTLS applied to hospitals is presented by Steve Nibbelink [5] in a case using RFID [6] [7] and RTLS and points out as main benefits the following aspects: patient and staff safety (by preventing patient elopement through integration with access control, video surveillance, and other electronic security and building technology.), hospital eficiency (reduced time and costs spent in locating assets, equipment, patients, and staff), hospital finance (with RFID and RTLS, organizations experience less theft), patient satisfaction (providing the very best in care and customer service to the patients, visitors, and staff improves patient experience and outcome). Another usage of RTLS in hospitals addresses the overcrowding of emergency departments [8] and refer the RTLS might offer a solution for this problem by gathering information about the workflow of hospital staff. Other similar contributions exist to track patients at a comprehensive stroke center [9], to manage patients at real time [10] or to represent a healthcare efficiency transformational initiative [11]. At a companies level, there has also been a considerable contribution with companies like IBM [12], PINCSolutions [1] or RapidModeling [2].

\section{Solution}

This section will describe our solution that, as we mentioned above, has the purpose to describe a solution for indoor asset tracking in a hospital environment. This solution will then be tested against most known and used materials in hospital buildings to evaluate the behavior of the system.

a) Overview and functionalities

The main purpose of this system is to allow track moving assets inside a hospital. The system has to be designed so anyone who accesses the computer of the floor can easily know two things: 1) where a specific equipment is and 2) the closer equipment of a given type (eg.: reanimation machine). Fig 1 represents an exam- ple of a floor plan showing a computer, where personnel access to get the position of equipments, and also several equipments distributed in the floor.

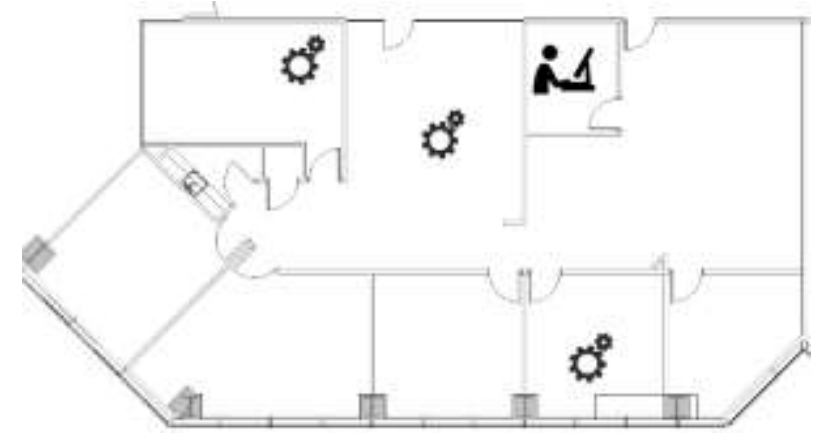

Fig. 1: Overview of the Purpose of the System, with Distributed Equipments and Central Computer.

b) Architecture and components

The architecture of this solution, shown in Fig 2, relies on beacons that communicate with Raspberry Pies (RP) over Bluetooth. On another hand, RP communicates with the server to send all data, that is processed and displayed in a web page.

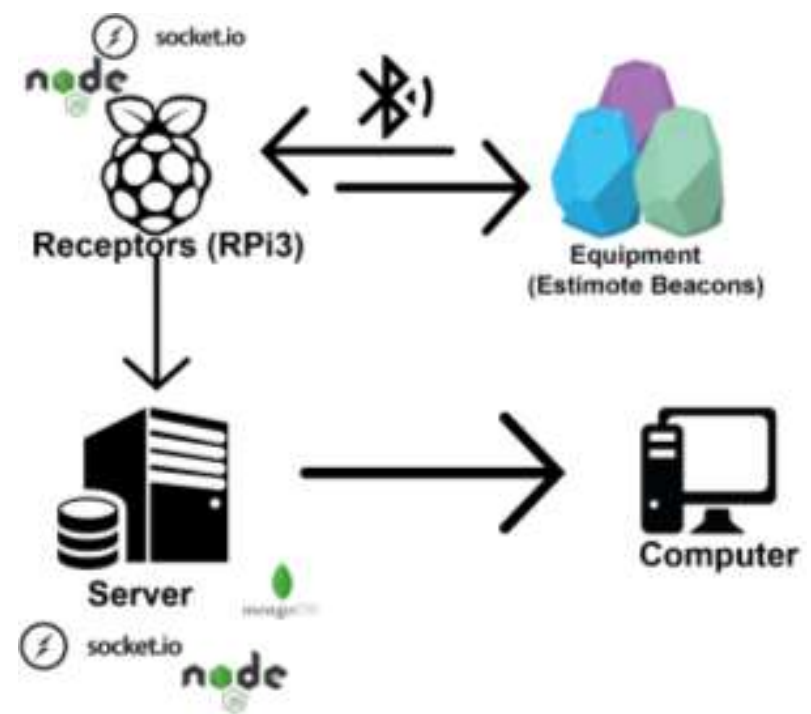

Fig. 2: Architecture of the System.

Server

We had some requirements for the server: it to be able to handle web sockets, be lightweight and versatile to be used for more applications. The choice was Node.JS which supports web sockets (with Socket.IO) and has plenty of libraries.

Beacons

The beacons used for this project where the Estimote Proximity Beacons. As previously mentioned, there are two type of packets: iBeacon and Eddystone. We chose the Eddystone packets for its telemetry packet that gives us an additional information although with Estimote, all beacons are manageable via web portal and mobile application.

Middleware

The middleware is responsible for the communication between the beacons and the server and this is done by Raspberry Pies. The Raspberry Pie 3 was the choice because it already includes Wi-Fi and Bluetooth and because there is a ARM version of Node.JS. To finish the setup of the middleware, we needed to configure BlueZ to get the Bluetooth to work in the Raspberry Pie and make Socket.IO to communicate with the server . We also used a Node.JS library (node-eddystone-beacon-scanner by Sandeep Mistry) in order to scan for the eddystone packets.

Database

The choice for the database was NoSQL and more specifically, MongoDB. MongoDB enables to build application faster, handle 
highly diverse data types and manage applications more efficiently at scale.

c) Implementation

In this section we describe details of the design of the solution Our approach, in order to achieve a better precision in the position of the equipments, was to map the floor of the hospital into coordinates (floors are treated independent from each other). With that mapping made, raspberries send beacons signals to the central server, where they are processed and allows personnel to search equipments in a backend.

Coordinate map definition

For each floor where we want to track assets, first we need a map of the floor in terms of coordinates. Before being able to track moving equipments, we defined the coordinates of fixed points that are the corners of each room and the position of the receptors (RP). From this point, we can start calculating the position of beacons (moving equipments). This calculation differs depending on the number of receptors that catch the beacon signal. The system supports three situations, of course with different levels of precision: three, two or one receptor catching the beacon signal. This signal, that is catched by the receptors, is the RSSI which stands for Received Signal Strengh Indicator and represents the measurement that indicates how well the beacon is perceived by the receptor. As this value is not the most accurate, we applied the Kalman filter to obtain a more precise value. In the next sections, we explain the application if the Kalman filter, how we calculate distance to the beacon and the three supported situations (three, two or one receptor catching the beacon signal).

Kalman filter

In order to get a smoother evolution of the distance of the beacon to the receptors, we implemented the Kalman filter [13], one of the most widely used methods for tracking and estimation due to its simplicity, optimality, tractability and robustness.

This algorithms takes a series of measurement over time containing noises and other inaccuracies and produce a variable that tend to be more accurate than just the raw values. Fig 3 shows values received from beacon signals with and without the Kalman filter, allowing us to conclude we would increase the precision of the system by using it.

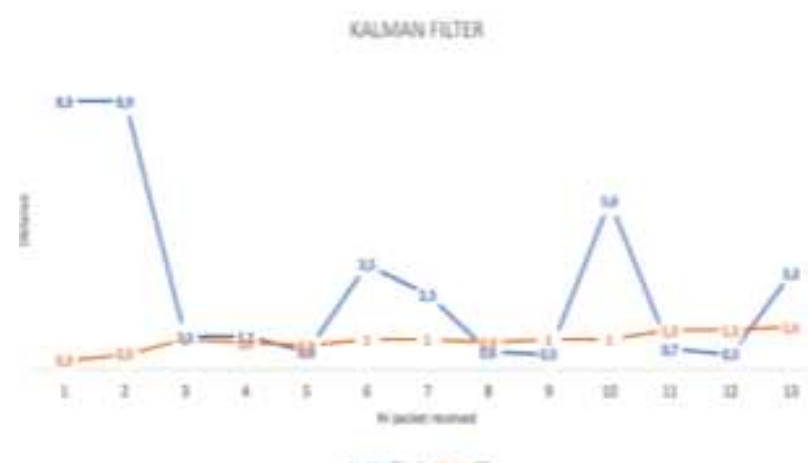

Fig. 3: Demonstration of Application of Kalman Filter to Received Signals from Beacons.

\section{Calculate distance}

In order to calculate the distance of the beacon to the RP, we use the formula of power regression. The necessary parameters are:

- $A_{0}$ : the txPower of the beacon (which is the physical power of the transmitted signal)

- RSSI: the value obtained from the Kalman filter

- $\mathrm{N}$ : the signal propagation, for indoor $\mathrm{n}=2$

- d: the value we want to obtain

With this information, we can apply the RSSI formula given by equation 1.

$$
A_{0}-R S S I=10 * n * \log (d)
$$

If we work the equation we can obtain the one described in equation 2 , which provides the value of the distance.

$$
d=10^{\left(\frac{A_{0}-R S S I}{10 * 2}\right)}
$$

The distance calculation presented is used in the next three situations that are incorporated in the system.

Three receptors catch the beacon

This is the most desired situation for the system to calculate the beacon position with more precision as it allows us to use trilateration. The trilateration only uses distances and coordinates to calculate an unknown point in opposite of triangulation that uses angles too. Trilateration uses the geometry of circles, spheres or triangles. Since we have the distance of the beacon from each receptor, we know that it will be in a circle around the receptor at the distance calculated. We also know that, given distances, each circle will intersect at some point and that is what we use. To find the intersection of the three circles, we must formulate the equation for the three circles surfaces and resolve it the two unknowns: $\mathrm{x}$ and $\mathrm{y}$. The unknown point is $(\boldsymbol{x}, \boldsymbol{y})$, the receptors are $\left(\boldsymbol{x}_{\boldsymbol{r} \boldsymbol{i}}, \boldsymbol{y}_{\boldsymbol{r} \boldsymbol{i}}\right)$ and we also know each distance $r_{i}$

$$
\begin{aligned}
& \left(x-x_{r 1}\right)^{2}+\left(y-y_{r 1}\right)^{2}=r_{1}^{2} \\
& \left(x-x_{r 2}\right)^{2}+\left(y-y_{r 2}\right)^{2}=r_{2}^{2} \\
& \left(x-x_{r 3}\right)^{2}+\left(y-y_{r 3}\right)^{2}=r_{3}^{2}
\end{aligned}
$$

Expanding each one gives us the folowing:

$x^{2}-2 x_{r 1} x+x_{r 1}^{2}+y^{2}-2 y_{r 1} y+y_{r 1}^{2}=r_{1}^{2}$

$x^{2}-2 x_{r 2} x+x_{r 2}^{2}+y^{2}-2 y_{r 2} y+y_{r 2}^{2}=r_{2}^{2}$

$x^{2}-2 x_{r 3} x+x_{r 3}^{2}+y^{2}-2 y_{r 3} y+y_{r 3}^{2}=r_{3}^{2}$

If we subtract the first with the second we get:

$\left(-2 x_{r 1}+2 x_{r 2}\right) x+\left(-2 y_{r 1}+2 y_{r 2}\right) y=r_{1}^{2}-r_{2}^{2}-x_{r 1}^{2}+$ $x_{r 2}^{2}-y_{r 1}^{2}+y_{r 2}^{2}$

And then subtract the second with the third:

$$
\begin{aligned}
& \left(-2 x_{r 2}+2 x_{r 3}\right) x+\left(-2 y_{r 3}+2 y_{r 3}\right) y=r_{2}^{2}-r_{3}^{2}-x_{r 2}^{2}+ \\
& x_{r 3}^{2}-y_{r 2}^{2}+y_{r 3}^{2}
\end{aligned}
$$

We now have a system of two equations with two unknown variables:

$A x+B y=C$

$D x+E y=F$

To know $x$ and $y$ we have:

$x=\frac{C E-F B}{E A-B D}$

$y=\frac{C D-A F}{B D-A E}$

After this, we have the position of the beacon, given by $\mathrm{x}$ and $\mathrm{y}$. Computationally, all of this is done on the server with algebra.js that allows us to compute equations in JavaScript.

Two receptors catch the beacon

In this case, we can make the calculus directly in JavaScript without the use of external libraries. We used a function named intersection that takes two receptors coordinates and two distances. We start by calculating the vertical and horizontal distances between the circle centers, then determine the straight-line distance between the centers. From here, what we want to obtain is the point where the line through the circle intersection points crosses the line between the circle centers. Next we present all the calculations to 
achieve this point which will be the beacon position, given two receptors catching its signal.

Function intersection(x0, y0, r0, x1, y1, r1) \{

var a, dx, dy, d, h, rx, ry;

var $\mathrm{x} 2, \mathrm{y} 2$;

$/ * \mathrm{dx}$ and dy are the vertical and horizontal distances between the circle centers. $* /$

$\mathrm{Dx}=\mathrm{x} 1-\mathrm{x} 0$

$\mathrm{dy}=\mathrm{y} 1-\mathrm{y} 0$;

$1 *$ determine the straight-line distance between the centers. */

$\mathrm{d}=$ Math.sqrt $((\mathrm{dy} * \mathrm{dy})+(\mathrm{dx} * \mathrm{dx}))$;

/* Check for solvability. */

If $(\mathrm{d}>(\mathrm{r} 0+\mathrm{r} 1))\{$

/* no solution. Circles do not intersect. */

Return false;

\}

if $(\mathrm{d}<$ Math.abs $(\mathrm{r} 0$ - r1 $))\{$

$/ *$ no solution. one circle is contained in the other */

Return false;

\}

$I^{*}$ 'point 2 ' is the point where the line through the circle

* Intersection points crosses the line between the circle

* Centers.

$* /$

$/ *$ determine the distance from point 0 to point $2 . * /$

$\mathrm{a}=((\mathrm{r} 0 * \mathrm{r} 0)-(\mathrm{r} 1 * \mathrm{r} 1)+(\mathrm{d} * \mathrm{~d})) /(2.0 * \mathrm{~d})$;

$/ *$ determine the coordinates of point 2 . */

$\mathrm{x} 2=\mathrm{x} 0+(\mathrm{dx} * \mathrm{a} / \mathrm{d})$

$\mathrm{y} 2=\mathrm{y} 0+(\mathrm{dy} * \mathrm{a} / \mathrm{d})$

$1 *$ determine the distance from point 2 to either of the

* Intersection points.

$* /$

$\mathrm{h}=$ Math.sqrt $((\mathrm{r} 0 * \mathrm{r} 0)-(\mathrm{a} * \mathrm{a}))$;

$/^{*}$ Now determine the offsets of the intersection points from

$*$ point 2 .

$*$ /

$\mathrm{rx}=-\mathrm{dy} *(\mathrm{~h} / \mathrm{d})$;

ry $=\mathrm{dx} *(\mathrm{~h} / \mathrm{d})$

/* determine the absolute intersection points. */

$\operatorname{var} \mathrm{xi}=\mathrm{x} 2+\mathrm{rx}$

var xi_prime $=\mathrm{x} 2-\mathrm{rx}$;

var yi $=$ y $2+$ ry;

var yi_prime = y2 - ry;

Return [xi, xi_prime, yi, yi_prime];

\}

One receptor catch the beacon

This case is rare but still can happen and must be considered. In this case, no coordinates are computed, both are set to -1 and we store in the database only the distance.

Back-end

Once the system can recognize the beacons position, which represent moving assets inside an hospital, we developed the backend so all authorized personnel can quickly find them. But the backend has also some configuration functionalities to make the system easily scalable. It is possible to create new building and add floors to it; and then add rooms to the floors. When adding rooms, the coordinates have to be defined as shown in Fig 4. Receptors also need to be inserted in the system, the room associated to it and also te coordinates so all algorithms and calculations described above to know the assets position can work. The beacons also need to be added to the system, with the Eddystone namespace and instance. Finally, the terminals also need to be added, which represent desktop computers where searched will be performed by the personnel. So the minimum distance can be calculated, the terminals also need to have coordinates.

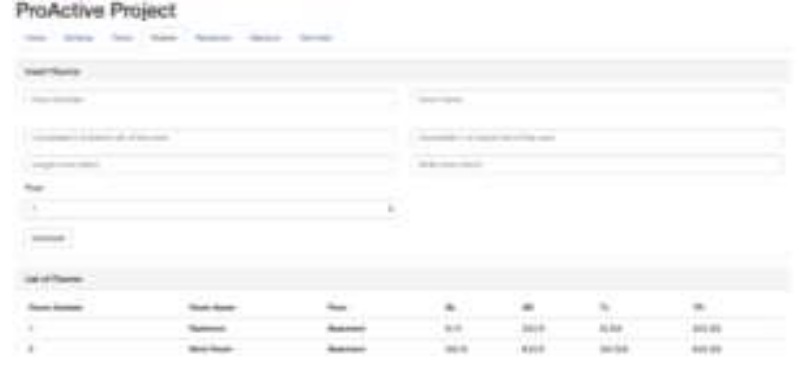

Fig. 4: Back-End Functionality to Add Rooms to Floors Including Coordinates Definition.

With all this information, searches can be done by personnel to track and find assets. Fig 5 shows the backend listing all equipments in floor 1 . In this case, there is one equipment named 'ice' (this is in fact the beacon name that would in production receive the name of the equipment) and the correspondent coordinates. The battery life is also presented so it can be replaced when necessary.

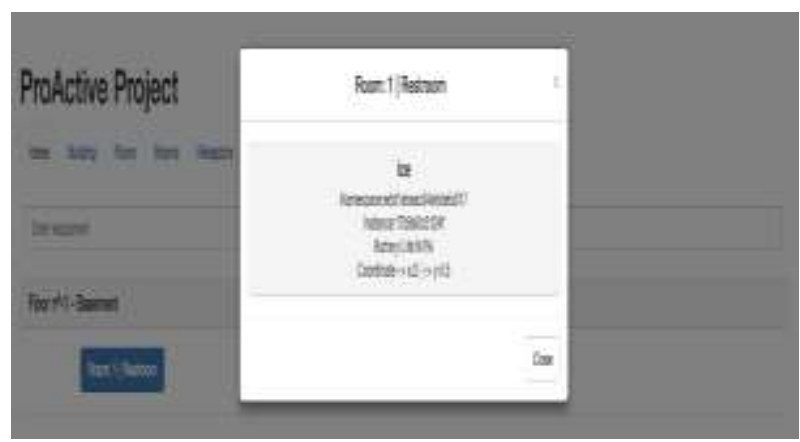

Fig. 5: List of All Equipment Found in Floor 1.

Fig 6 shows a specific search, for a equipment with a given name. In this case is 'ice' but it could be a reanimation machine or any other suitable name for the equipment. After searching for the device, the closer location is presented which in this case in the restroom of the basement floor.

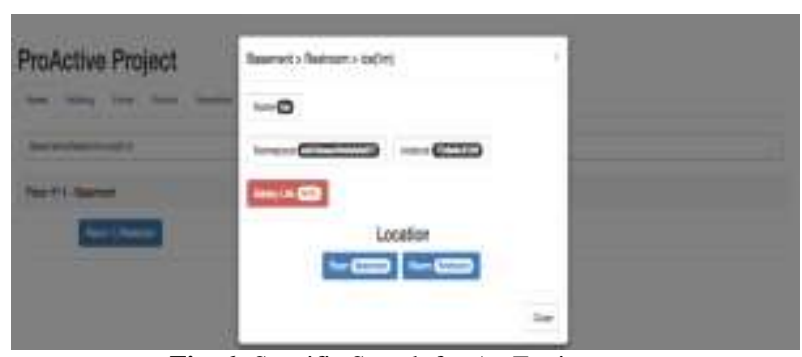

Fig. 6: Specific Search for An Equipment.

\section{Evaluation}

The evaluation of this system is fundamental to ensure it has a proper behaviour in several scenarios inside a hospital. In concrete, we refer to the several materials that buildings are made of and the influence they have on beacons signals. Beacons, being part of the Radio Frequency family, are sensible to materials just like Wi-Fi is. Some of the situations that are likely to happen in hospitals and that we contemplate in this evaluation are:

- Given a hospital room, can one bed/machine position be confused with another bed the floor below (making it a different room?) Certain materials block or help to propagate a signal. In a big building, this can be a problem in a floor level to identify which beacons are in this floor and do not confuse with another floor.

- Given a hospital room, can one bed/machine position be confused with another bed the other side of the wall (making it a 
different room?). This aspect has to do with the influence of materials and next we will present the tests made.

- Given a hospital room, can curtains or people obstruct the signal and prevent the detection of an equipment? This aspect is also covered by the influence of materials on detecting and catching signals.

a) Beacons between floors

Differenciation in the same floor is handled by the calculation of the coordinates and since the calculations does not involve the $\mathrm{z}$ axis, we need some additional information. In our system, each beacon is identified by a namespace and an instance. The namespace is a general tag that could be associated with the family the equipment represent, in our case the hospital. We this in mind, the instance is what identify uniquely a beacon. We need to represent the floor and still uniquely identify the beacon; the instance is represented by a 12 character string long that offers a lot of possibilities to associate identificators with. If we take the first character as the representation of the floor, we end-up with 11 characters that still offers plenty of identificators. If we use the hexadecimal base number to represent the ids, we go from $2.8147498 \mathrm{e}+14$ for a 12 character id to $1.7592186 \mathrm{e}+13$.

b) Placement of the receptors

In order to get the most area covered and to match the characteristics of the RSSI values and coordinate system, the placement for the receptors have to be in a high place. Covering them in a surface where people can have access too isn't the best idea. The best solution is to place them on the ceiling, in the center of the room.

c) Evaluation of the RSSI values

Being a radio signal, RSSI values are highly affected by materials or other signals. A list of materials and their level of interference is shown in Table 1.

Table 1: Interference Potential of Several Material Types

\begin{tabular}{ll}
\hline Interference potential & Material types \\
\hline Low & Wood, synthetic materials and glass \\
Medium & Bricks and marble \\
High & Plaster, concrete and bulletproof glass \\
Very high & Metal, water \\
\hline
\end{tabular}

Note that the water type is equivalent to the human body due to his composition of $70 \%$.

Modification through materials

To test the interference of materials on our solutions and understand the adjustments we would need to do, we used several materials at a 2 meters distance between the receptor and the beacon. For the tests, the following materials were used: wood, brick, concrete low thickness $(<5 \mathrm{~cm})$ and concrete high thickness $(>30 \mathrm{~cm})$, metal low thickness $(<5 \mathrm{~cm})$ and metal high thickness $(>30 \mathrm{~cm})$, crowd (water). The concrete and metal scenario had to be split in two categories to represent better the impact that they have, since there potencial in interfering with the signal is high.

The first test, whose results are shown in Fig 7, show that the lowest values of RSSI are obtained with people around the beacon (water) and concrete/metal high thickness.

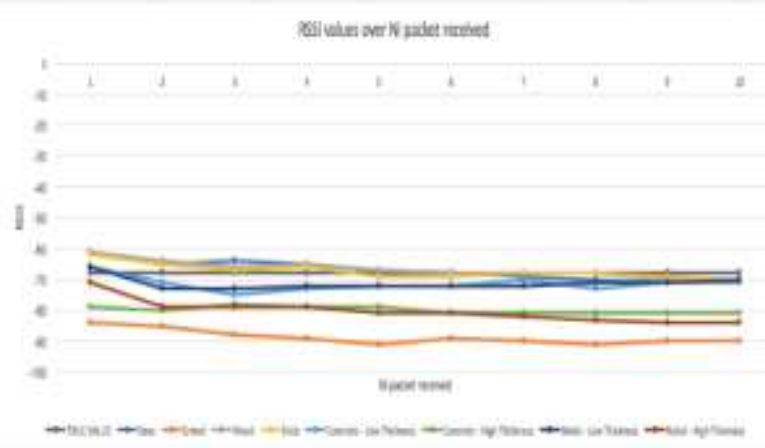

Fig. 7: RSSI Values over Time for Each Material

Another test was made regarding the distance signals are received with the interference of the materials. Results are shown in Fig 8 and, once again, people represent the highest interference, resulting in a lower number of packets received.

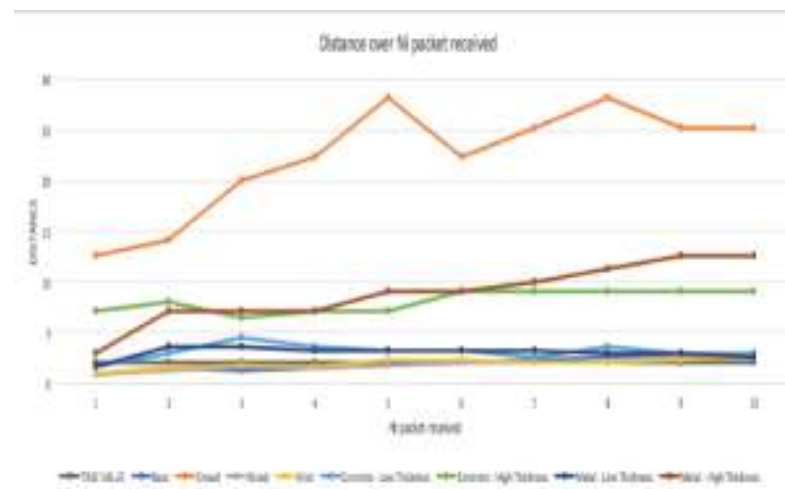

Fig. 8: Distance over Time for Each Material.

Modification through other signals

Bluetooth belongs to the $2.4 \mathrm{GHz}$ bandwith, making it another equipment using it. The most common signal that we can find in every corner and can interfere with the beacons that belongs to the same bandwith is the Wi-Fi signal. Correctly used, there is not any problem with the co-existence of both signals. The best practice for WiFi configuration is enabling WiFi on one of the following channels: $1,6,7,8,9,10,11,12$. Other channels $(2,3,4,13,14)$ might cause interference with Bluetooth signal and it's best to avoid them.

These tests allowed us to conclude that the system has a normal behavior in moments where visits are not in the hospital as the medical staff does not represent a crowd and will not obstruct the signal at the point of avoiding detection. For the moments of visits, and to overcome the obtained results with a crowd, and acoording to the design of our system, we recommend the placement of several raspberries in rooms that are larger so a better reception of signal from the beacons is possible. Regarding concrete, another material that did not obtain good results in the tests, it will only be a problem in buildings that have this materials between rooms.

\section{Conclusion}

In this paper we presented a server-based positioning system using BLE for tracking assets in hospitals. Certain equipment can be crutial and a matter of life or dead and need to be found in a matter of seconds. The existence of a system that can be used by hospital personnel to search for equipment is a huge benefit for the institution and everyone that trust it. We presented a solution using Bluetooth low energy technology, that communicate with Raspberry Pie which sends data to a server for processing so it can be visualized in a backend. The main purpose is for personnel, using a desktop computer, can easily and quickly find a equipment.The solution requires each floor to be configured through a backend and also the position of the Raspberry Pie so coordinates can be calculated.

We presented in detail the algorithms for calculating the distance between Raspberry Pie and beacon and also the beacon final coordinates. Finally we made some tests regarding the interference of materials having reached the conclusion that water (people) and concrete high thickness are the worst scenarios for the system we designed. Not all building are made of concrete high thickness so in this cases confusion between adjacent rooms will not be a problem. People are in fact the biggest problem but we believe this will only happen in visit moments and if the equipment is blocked by all of them. Even so, we suggest having more than one receptor in bigger rooms so RSSI signals can be caught with better quality and contribute to a better behavior of the global system, with higher precision in the calculated coordinates. 


\section{References}

[1] PINC, "Mobile Asset Tracking with Real-Time Location Systems (RTLS): The Real-Time Visibility Enabler for a Global Supply Chain.

[2] N. E. Lee, "RTLS and the Built Environment RTLS and the Built Environment," 2010.

[3] J. A. Fisher and T. Monahan, "Evaluation of real-time location systems in their hospital contexts," Int. J. Med. Inform., vol. 1, pp. 705-712, 2012.

[4] A. Simmons, "Improved OR Patient Flow through RTLS-Based Visual Analytics," in HIMSS, 2016.

[5] S. Nibbelink, "A primer on RFID and RTLS technologies for healthcare facilities," 2012.

[6] "How to Choose the Right RFID System: A Step-by-Step Guide for Providers of Health Care," RFID J., 2011.

[7] S. Pique, "RFID, RTLS and NFC in healthcare," 2015.

[8] R. M. Jochem, "Accepting and engaging with a real-time location system in a hospital context Accepting and engaging with a realtime location system in a hospital context," Eindhoven University of Technology, 2017.

[9] A. Prater and M. Bowen, "Using a real-time location system to track patients at a comprehensive stroke center: a process improvement project," Am. Coll. Radiol.

[10] N. Siva and A. Rajesh, "RFID-Based Hospital Real Time Patient Management System,” Int. J. Comput. Trends Technol., vol. 3, pp. 509-517, 2012.

[11] Z. Smith, "VA Real Time Location System (RTLS) Overview," New Engl. Soc. Clin. Eng., 2014.

[12] G. Buchan, "Real Time Location Services \& Asset Management," IBM, 2009.

[13] S. J. Julier and J. K. Uhlmann, "A New Extension of the Kalman Filter to Nonlinear Systems,” SPIE, vol. 3068, pp. 182-193, 1997. 